\title{
Cooperation in the prisoner's dilemma as a function of interpersonal distance
}

\author{
JOHN SENSENIG and THOMAS E. REED \\ University of Kentucky, Lexington, Ky. 40506 \\ and NIMH Clinical Research Center, Lexington, $\mathrm{Ky} .40507$ \\ and \\ JEROME S. MILLER \\ NIMH Clinical Research Center, Lexington, Ky. 40507
}

The prisoner's dilemma game was played by Ss seated either physically close together or separated by a substantial physical distance. With greater distance, there were significantly fewer mutually cooperative choices, smaller earnings, and greater differences in outcome between the two players. Mutual cooperation among Ss in the far condition remained relatively infrequent throughout the trial sequence and extinguished completely in later trials.

Interest in the prisoner's dilemma (PD) game stems primarily from the inherent conflict between individual and mutual gain. As an analogue to a broad class of real-life conflict situations, the PD tempts each player to competitive responses in seeking individual gain or comparative success in relation to the other participant. If both players adopt a competitive or exploitative strategy, both lose. Mixed cooperative-competitive play by the two participants results in variable outcomes. Profit for both players, however, is assured if each responds cooperatively.

Among the known variables that influence the level of cooperative play in the PD are such factors as the cooperative index of the matrices employed (Terhune, 1968), the strategy or sequential responses presented to the S (Oskamp, 1970; Rapoport \& Chammah, 1965), and individual differences in motives and personality (Deutsch, 1960; Terhune, 1968). Social perception factors also have been shown to influence the degree of cooperative play in PD, e.g., the amount of information about the other player (Marlowe, Gergen, \& Doob, 1966) and the S's perceptions of the interaction and his expectations concerning the other participant's likely behavior (Kelley \& Stahelski, 1970).

The present investigation of cooperation in the $\mathrm{PD}$ draws upon suggestive research on interpersonal distance, i.e., the physical distance between two persons. In particular, this literature has indicated that the distance between persons affects perceptual and behavioral processes of interpersonal interaction. Conceptualization of the communicative processes involved in varying distances has been offered by Hall (1966). Patterson \& Sechrest (1970) found that impression formation varied as a function of interpersonal distance. Albert \& Dabbs (1970) showed that distance influenced persuasion. More directly related to the $P D$ game's cooperative and competitive strategies or sets, Sommer (1969) has shown that people chose seats further from others when anticipating competition than when expecting to engage in a cooperative task. Our study tested the complementary proposition, i.e., that higher cooperation is attained when persons are seated closer to each other. METHOD

Twenty dyads of male Ss, all residents at the NIMH Clinical Research Center at Lexington, Kentucky, received tape-recorded instructions prior to playing 50 trials of PD. Personal distance was varied by seating two Ss either at the same table (close condition) or at widely separated tables (far condition). The distances involved were approximately 3 and $20 \mathrm{ft}$, respectively. Visual aspect between players was the same in both conditions, i.e., at a right angle with no visual barrier. Each $\mathrm{S}$ had before him an electronic console which sequentially displayed a randomized set of matrices with a common index of cooperation of .5 (Terhune, 1968). Monetary payoff based upon the number of points accumulated was promised, and each console displayed the cumulative point totals for both the $S$ and the other player. A S could choose to cooperate or to defect by pushing one of two buttons on his console. Following each trial, automatically, an arrow on the console pointed to the joint outcome, the cumulative point totals were updated, and a new matrix was displayed. When the trials were completed, arrangements were made for the Ss to receive payment for the points they had each accumulated.

\section{RESULTS}

A typical measure of cooperation used in the PD game is the mean outcome within dyads. Applying this comparison reveals that the mean individual outcome of 160.75 $(s=14.79)$ earned points in the near condition is significantly higher than the mean outcome of 115.35 $(s=25.79)$ earned points obtained in the far condition $(t=2.06, \mathrm{df}=38$, $\mathrm{p}<.05)$. Another related analysis utilizing the mean difference in outcome between the winner (player with the highest outcome) and the loser within each dyad reveals a similar pattern. For the near condition, the average mean difference between players within dyads was 65.90 $(s=54.66)$, which is significantly less than the mean difference of 160.70 $(s=100.19)$ in the far condition 1 $(t=2.63, d f=18, p<.01)$.

A third measure of cooperation was obtained by examining the kinds of actual responses made by the players. Since on a given trial each player can choose either to cooperate or to defect, there are three possible types of response combinations for a pair of Ss on each trial: both players may cooperate (CC), both may defect (DD), or their choices may be mixed (CD). If the latter type of responses (CD) are eliminated on the basis of their ambiguity, a comparison can be made utilizing the frequency of occurrence of $\mathrm{CC}$ as opposed to $\mathrm{DD}$ response patterns. Analyses of the differences showed that the CC response pattern occurred more frequently $(t=5.14, \quad d f=18$, $\mathrm{p}<.001)$ in the near condition $(\bar{X}=9.7, s=11.01)$ than in the far condition $(\bar{X}=2.7, s=2.75)$. The DD pattern occurred with greater frequency $(t=2.56, \mathrm{df}=18, \mathrm{p}<.05)$ in the far condition $(\overrightarrow{\mathrm{X}}=30.1$, $s=7.14$ ) than in the near condition $(\bar{X}=23.4, s=12.79)$. Out of the total of 500 trials, Ss in the far condition made mutually cooperative responses (CC) on only 27 trials, or less than $6 \%$ of the time. Comparable figures for the near condition show that the CC pattern occurred on 97 trials, or $19 \%$ of the time. The DD pattern occurred with frequencies of 234 and 301 in the near and far conditions, respectively.

Figure 1 shows the frequency of occurrence of the $\mathrm{CC}$ pattern over trials for both experimental groups ( $N$ $=10$ dyads in each condition). Each of the 10 blocks contains five trials. It can be seen that there is no overlap in the two curves and that the CC level for the near condition is initiated earlier and remains consistently higher than the CC level for the far condition. The highest level of $\mathrm{CC}$ in the near condition was during the sixth block of trials, while the highest level in the far condition was during the seventh block. It can also be seen that the $\mathrm{CC}$ level in the near group regressed to the 


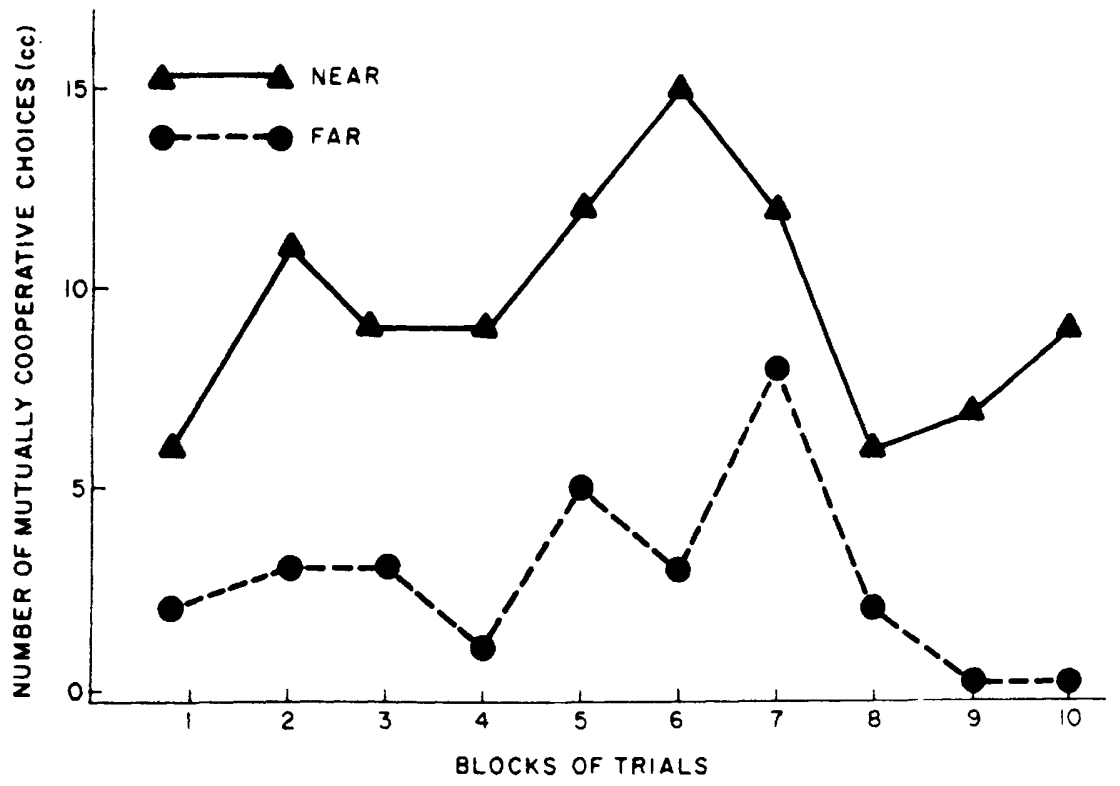

Fig. 1. Number of mutually cooperative choices in blocks of five trials. $(\mathrm{N}=$ 10 dyads in each condition.)

starting level only once (Block 8 ) with an increasing trend in Blocks 9 and 10. In contrast, the $\mathrm{CC}$ level in the far condition returned to or fell below its initial level four times, and dropped to zero $\mathrm{CC}$ responses in the last two blocks of trials, i.e., no $\mathrm{CC}$ responses in 100 opportunities.

\section{DISCUSSION}

The finding of greater cooperation in the near condition provides evidence for the proposition that closer seating arrangements lead to more cooperation. This result is also consistent with related work, e.g., Hall's (1966) emphasis upon cultural norms' guiding behavioral interaction within certain zones, Mehrabian's $(1968 \mathrm{a}, \mathrm{b})$ finding that closeness leads to increased positive feelings-provided one is not too close, and Sommer's (1969) demonstration that Ss choose to sit closer when anticipating cooperative interaction rather than competition.

The present result is also consistent with Newcomb's (1947) discussion of "autistic hostility," i.e., that there is a greater likeli hood of misunderstanding, hostility, and results, no further speculations will be made. It will suffice to say that the obtained results suggest that not only does distance influence initial interaction, but that this influence ma become increasingly determinative with continued interaction.

\section{REFERENCES}

ALBERT, S., \& DABBS, J. M., JR. Phy sical distance and persuasion. Journal of Personality \& Social Psychology, 1970. $15,265-270$.

DEUTSCH, M. Trust, trustworthiness, and the $F$ scale. Journal of Abnormal \& Social Psychology, 1960,61,138-140.

HALL, E. T. The hidden dimension. New York: Doubleday, 1966.

KELLY, H. H., \& STAHELSKI, A. J. Social interaction basis of cooperators' and competitors' beliefs about others. Journal of Personality \& Social Psychology, 1970. $16,66-91$.

MARLOWE, D., GERGEN, K. J., \& DOOB. A . N. Opponent's personality. expectation of social interaction, and interpersonal bargaining. Journal of Personality \& Social Psychology, 1966, 3 . 206-213.

MEHRABIAN, A. Inference of attitude from the posture, orientation, and distance of a communicator. Journal of Consulting \& Clinical Psychology, 1968a, 32, 296-308.

possible exploitation when lack of communication leads to increased interpersonal "distance." Particularly striking was the total absence of mutually cooperative responses among Ss in the far condition during the last two blocks of trials. It is clear that Ss in this condition had given up any attempt at cooperation and were responding competitively even in the face of increasing personal cost. It is possible that the lower outcome level, which developed early in the sequence for players in the far condition, led to the absence of cooperative responses observed in the final blocks of trials. Another possibility is that greater mean difference between the winner and loser with the dyads in the far condition served as a comparative reward to the player within the higher payoff and strengthened the competitive tendency. It is noteworthy, however, that this same extinction process did not occur in the near condition, even though these Ss were also having difficulty in mutual cooperation. Since this study did not at tempt to assess mediating mechanisms that would explain the
MEHRABIAN, A. Relationship of attitude to seated posture, orientation, and distance. Journal of Personality \& Social Psychology, 1968b, 10, 26-30.

NEWCOMB, T. M. Autistic hostility and social reality. Human Relations, 1947,1 , 69-86.

OSKAMP, S. Comparison of strategy effects in the prisoner's dilemma and other mixed motive games. Proceedings: 78 th Annual Convention, American Psychological Association, 1970. 433-434.

PATTERSON, M. L., \& SECHREST, L. B. Interpersonal distance and impression formation. Journal of Personality, 1970 , $38,161-166$

RAPOPORT, A. \& CHAMMAH A. M. Prisoner's dilemma. Ann Arbor: University of Michigan Press, 1965.

SOMMER, R. Personal space: The behalio basis of design. Englewood Cliffs, N.J : Prentice-Hall, 1969.

TERHUNE, $K, W$, Motives, situation, and interpersonal conflict within prisoner's Psychology Monograph Supplement. 1968, 8(3, Pt. 2).

$$
\text { NOTE }
$$

1. This relatively high difference between the winner and the loser in the far condition reflects the fact that the matrices included negative entries and it was therefore possible for Ss to obtain negative point totals. This, in fact, turned out to be the case for several Ss, most of whom, it is suggestive to note. were in the far condition. dilemma. Journal of Personality \& Social 